\title{
Abundance analysis of DAZ white dwarfs
}

\author{
Adéla Kawka*, Stéphane Vennes*, František Dinnbier ${ }^{\dagger}$, Helena Cibulková ${ }^{\dagger}$ \\ and Péter Németh* \\ *Astronomický ústav, Akademie věd České republiky, Fričova 298, CZ-251 65 Ondřejov, Czech \\ Republic \\ ${ }^{\dagger}$ Faculty of Mathematics and Physics, Charles University, V Holešovičkách 2, CZ-180 00 Praha, \\ Czech Republic
}

\begin{abstract}
We present an abundance analysis of a sample of 33 hydrogen-rich (DA) white dwarfs. We have used archival high-resolution spectra to measure abundances of calcium, magnesium and iron in a set of 30 objects. In addition, we present preliminary calcium abundances in three new white dwarfs based on low-dispersion spectra. We investigate some abundance ratios $(\mathrm{Mg} / \mathrm{Ca}$, $\mathrm{Fe} / \mathrm{Ca}$ ) that may help uncover the composition of the accretion source.
\end{abstract}

Keywords: white dwarfs - stars: abundances

PACS: 97.20.Rp, 97.10.Tk

\section{INTRODUCTION}

Most white dwarfs possess a hydrogen-rich atmosphere (DA), with the remainder having a helium-rich atmosphere. Heavy elements are expected to sink below the photosphere because of the high surface gravity leaving either a pure-hydrogen or pure-helium atmosphere. However, a significant fraction of white dwarfs show traces of elements heavier than helium. In hot white dwarfs $(>20000 \mathrm{~K})$ radiative levitation can support metals against gravitational settling (Chayer et al. 1995). But in cooler white dwarfs an external source is required to feed the atmosphere with heavier elements. Scenarios explaining the presence of these elements in white dwarf atmospheres include accretion from the interstellar medium (Dupuis et al. 1993; Koester \& Wilken 2006), from unseen companions (Debes 2006), or from asteroidal/planetary material in circumstellar debris discs (Graham et al. 1990; Debes \& Sigurdsson 2002; Jura 2003).

Approximately one quarter of cool DA white dwarfs show the presence of metals in their spectra (DAZ; Zuckerman et al. 2003), and out of these approximately $20 \%$ have a mid-infrared excess that is consistent with a circumstellar disc (Farihi et al. 2009). Preliminary population statistics (Farihi et al. 2009) showed that white dwarfs with a higher calcium abundance were more likely to show IR excess than those with a lower abundance. Using Spitzer observations of several DAZ white dwarfs and their heliumrich counterparts (DZ), Farihi et al. (2010) showed that the size of a debris disc can vary, and that some debris discs may be narrow enough to remain undetected.

We present abundance measurements for a sample of known and new DAZ white dwarfs. The analysis of known DAZ white dwarfs was prompted by the discovery of magnesium and other heavy elements in the heavily polluted white dwarf GALEX J1931+0117 (Vennes et al. 2010) and the lack of detection of metals other than 


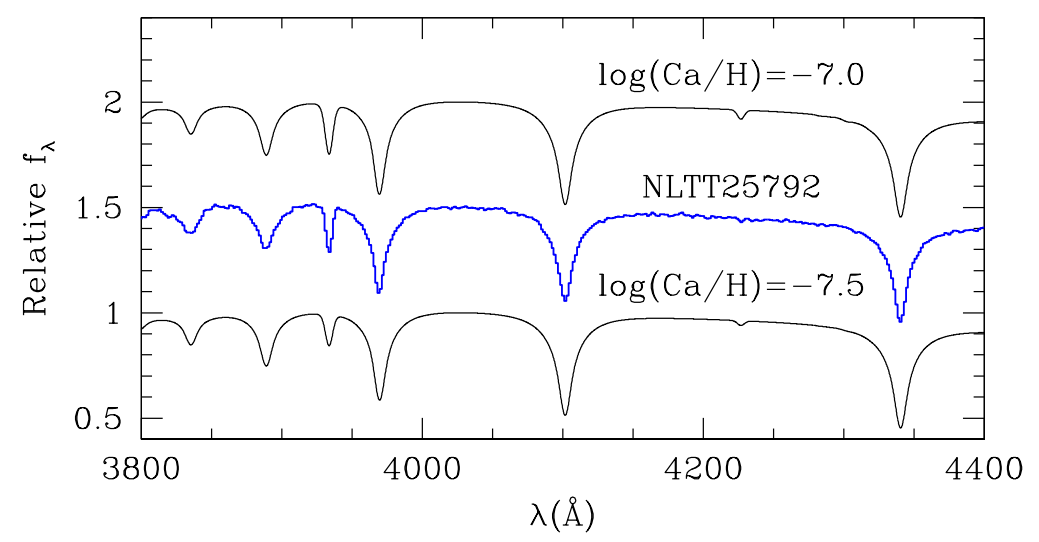

FIGURE 1. FORS 1 spectrum of NLTT25792, showing the Ca II $\lambda 3933.7 \AA$ and Ca I $\lambda 4226.7 \AA$ lines, compared to synthetic spectra at $T_{\text {eff }}=8160 \mathrm{~K}, \log g=8$ and $\log (\mathrm{Ca} / \mathrm{H})=-7$ and -7.5 .

calcium in other DAZ white dwarfs. We therefore initiated a search for heavy elements in DAZ white dwarfs using archival and new spectroscopy. The new DAZ white dwarfs were identified as part of our spectroscopic observations of white dwarf candidates in high-proper motion catalogues (NLTT, LSPM).

\section{ATMOSPHERIC PROPERTIES}

We have extracted UV-Visual Echelle Spectrograph (UVES) and High Resolution Echelle Spectrograph (HIRES) exposures for a sample of DAZ white dwarfs from the European Southern Observatory (ESO) and the Keck archives, respectively. We have also identified three new DAZ white dwarfs in the New Luyten Two-Tenths (NLTT) catalogue. The low resolution spectra obtained using the $4 \mathrm{~m}$ telescope at Cerro Tololo Inter-American Observatory (CTIO) or using the VLT-Kueyen telescope and FORS1 showed that NLTT 6390, NLTT 23966 and NLTT 25792 are cool DAZ white dwarfs. Calcium in the new DAZ white dwarfs is clearly photospheric and not interstellar.

We determined the effective temperature and surface gravity of the white dwarfs by fitting the Balmer line spectra with synthetic spectra for pure hydrogen models. Kawka \& Vennes (2006) summarizes the model atmosphere calculations. The grid covers effective temperatures from $4500 \mathrm{~K}$ to $50000 \mathrm{~K}$ and surface gravities from $\log g=5.75$ to 9.50 . The model spectra were convolved with the instrumental resolution. The UVES spectra were binned to $1 \AA$ before fitting the Balmer lines with model spectra. Because the HIRES echelle spectra are not flux calibrated, we obtained a Sloan Digital Sky Survey (SDSS) spectrum for WD1455+298 and fitted the Balmer lines to determine the effective temperature and surface gravity. The atmospheric parameters for WD0208+396 were taken from Gianninas et al. (2005).

We searched for the presence of metals in the high-resolution spectra. Several lines of calcium, magnesium and iron were detected, but in the present analysis we restricted the abundance determinations to Ca II $\lambda 3934 \AA, \operatorname{Mg}$ I $\lambda 5184 \AA, \operatorname{Mg}$ II $\lambda 4481 \AA$, and Fe I $\lambda 3581 \AA$. We calculated a set of LTE spectra with varying calcium, magnesium and iron 


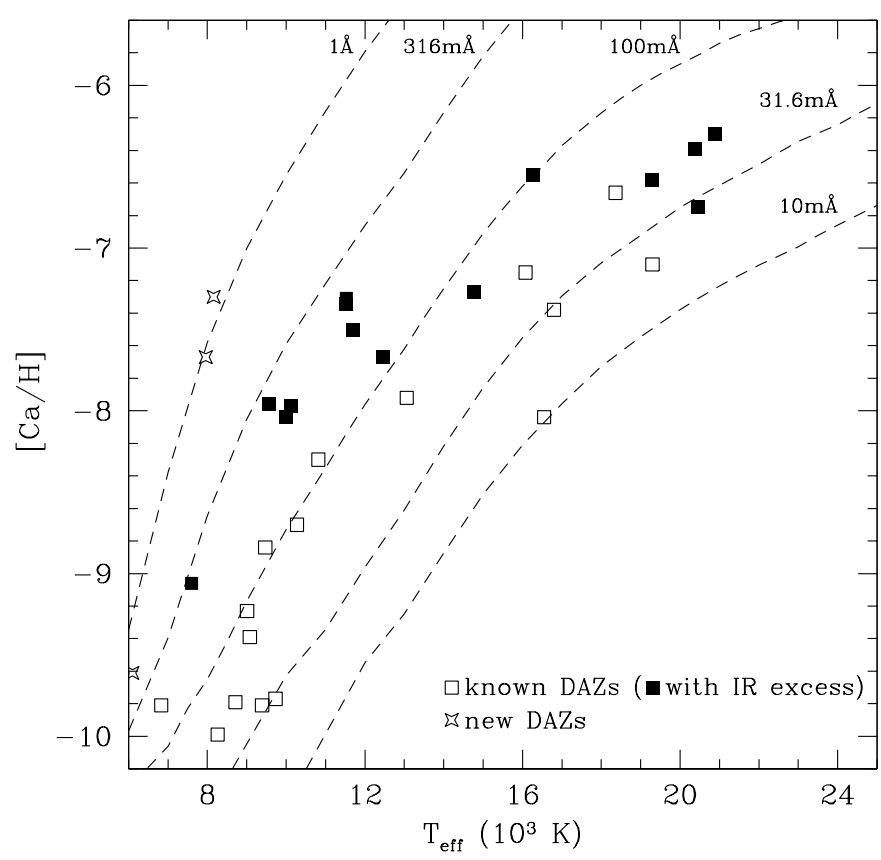

FIGURE 2. Abundance of calcium versus effective temperature. Filled squares are white dwarfs with confirmed infrared excess, and open squares are white dwarfs either without infrared excess or that have not been checked for infrared excess. The dashed lines are curves of constant equivalent widths for Ca II $\lambda 3933 \AA$.

abundances at $\log g=8$ and with temperatures ranging from 6000 to $25000 \mathrm{~K}$. We then computed synthetic equivalent widths for the selected lines within windows of $\Delta=10 \AA$ for Ca II and Mg II, $\Delta=8 \AA$ for $\mathrm{Mg}$ I, and $\Delta=6 \AA$ for Fe I.

For NLTT 25792, we estimated the calcium abundance by fitting synthetic spectra to the Ca II $\lambda 3934 \AA$ line profile. Figure 1 shows the observed FORS 1 spectrum compared to two model spectra with $\log (\mathrm{Ca} / \mathrm{H})=-7.0$ and -7.5 .

For the remaining stars we determined the abundances by fitting the measured equivalent widths with the synthetic equivalent widths. Since we did not have a spectrum covering $\mathrm{Ca}$ II $\mathrm{H}$ and $\mathrm{K}$ lines for WD0208+396 we used the abundance determined by Zuckerman et al. (2003). The atmospheric parameters and abundances for GALEX J1931+0117 were taken from Vennes et al. (2010).

Calcium is the most frequently detected element in DAZ white dwarfs (Zuckerman et al. 2003; Koester et al. 2005) and is a prevalent metallicity indicator. Figure 2 shows the calcium abundance versus effective temperature measurements in our sample. Stars with confirmed IR excess indicative of a debris disc are shown with filled squares, and stars that either do not have IR excess or have not been observed in the mid-IR are shown with open squares. The calcium abundances in the new cool DAZ white dwarfs appear to be above average within their temperature ranges.

The first detection of magnesium in a H-rich white dwarf was in EG102 (Holberg et al. 1997). Most white dwarfs with magnesium also show an IR excess. Magnesium was later 


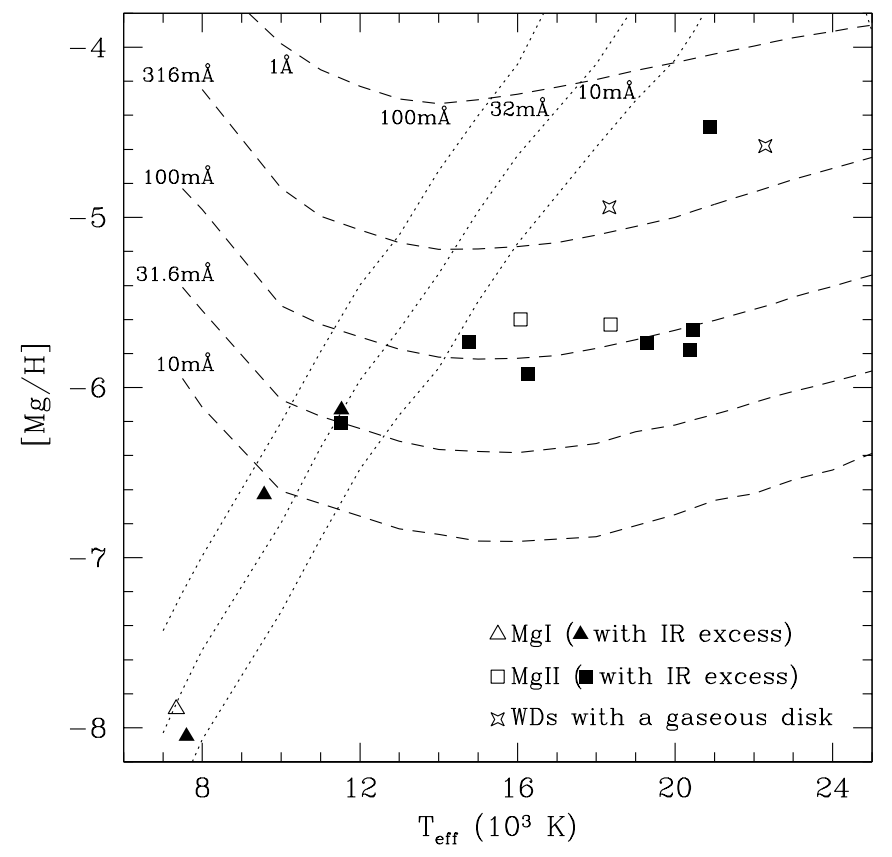

FIGURE 3. Abundance of magnesium versus effective temperature. Filled symbols are white dwarfs with confirmed infrared excess, and open symbols are white dwarfs either without infrared excess or that have not been checked for infrared excess. The dashed and dotted lines are curves of constant equivalent widths for Mg II $\lambda 4481 \AA ̊$ and Mg I $\lambda 5184 \AA$, respectively.

detected in several DAZ white dwarfs studied by Zuckerman et al. (2003) and in the two white dwarfs (SDSS J1043+0855 and SDSS J1228+1040) with a gaseous disc (Gänsicke et al. 2007). Four of our stars have been analyzed by Zuckerman et al. (2003) and our measured magnesium abundances are in agreement. In the case of WD2326+049, we measured an abundance $\log (\mathrm{Mg} / \mathrm{H})=-6.2$ somewhat lower than their value of -5.8 . We measured the equivalent widths of $\mathrm{Mg}$ I and $\mathrm{Mg}$ II lines in UVES and HIRES spectra of WD2326+049 and the corresponding abundance varied between -6.0 and -6.25 .

Figure 3 shows the magnesium abundance measurements versus effective temperatures. The stars with IR excess are shown with filled symbols and stars without IR excess with open symbols. The figure also shows the magnesium abundance determined by Gänsicke et al. (2007) for two white dwarfs with a gaseous disc. For WD2346+049, abundance measurements determined from both $\mathrm{Mg}$ I and $\mathrm{Mg}$ II lines are shown.

We also searched for iron lines and report its detection in five white dwarfs. Weak Fe I and $\mathrm{Fe}$ II lines are difficult to detect and therefore require high signal-to-noise spectra. Figure 4 shows the abundance of iron versus temperature, where all stars except two (WD0208+396, WD1202-232) have confirmed IR excess (filled symbols). The plot also includes GALEX J1931+0117 which is hot enough to display Fe II lines (triangle).

Table 1 lists the atmospheric properties and abundances of calcium, magnesium and iron in the DAZ white dwarfs. The parameters of GALEX J1931+0117 are also 


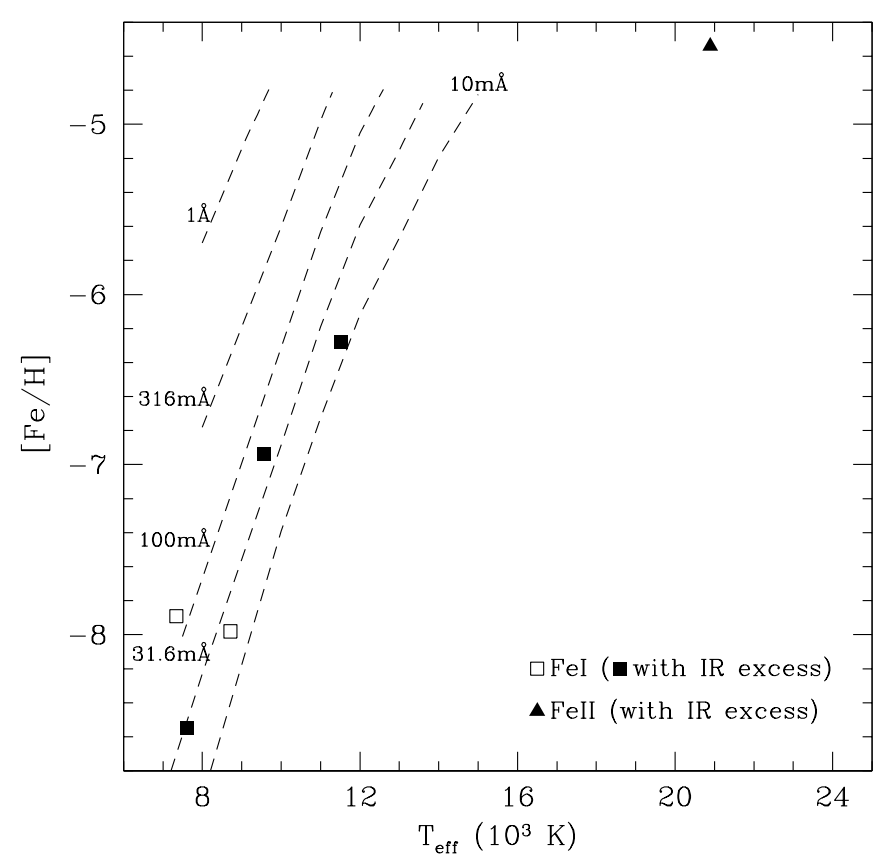

FIGURE 4. Abundance of iron versus the effective temperature. Filled squares are white dwarfs with confirmed infrared excess, and open squares are white dwarfs either without infrared excess or that have not been checked for infrared excess. The dashed lines are the equivalent widths for the Fe I $\lambda 3581 \AA$ line.

tabulated.

As first reported by Farihi et al. (2009), white dwarfs with a higher calcium abundance are more likely to harbour a debris disc than those with a lower abundance. We also found that the majority of white dwarfs with magnesium $(\approx 75 \%)$ and iron $(\approx 66 \%)$ in their atmosphere show an IR excess representative of a debris disc. Figure 5 shows measured $\mathrm{Mg} / \mathrm{Ca}$ and $\mathrm{Fe} / \mathrm{Ca}$ abundance ratios. The stars without a detectable IR excess were observed with Spitzer, but are otherwise similar to the other DAZ white dwarfs. However, their discs may be too narrow to be detectable (Farihi et al. 2010).

\section{CONCLUSIONS}

Spectroscopic studies of accreted material can help reveal the chemical composition of the accretion source. In our sample of white dwarfs, magnesium was detected in 12 white dwarfs (including GALEX J1931+0117) with temperatures ranging from $\approx 7000$ to $20000 \mathrm{~K}$. Iron was detected in five cooler white dwarfs $\left(T_{\text {eff }}<12000 \mathrm{~K}\right)$.

Abundance levels in the atmosphere depend on both the diffusion time-scales of the element and the accretion rate of individual elements (Koester \& Wilken 2006). Although diffusion time-scales are longer in cooler stars, the resulting abundances are lower for a given accretion rate because of the increased mass of the convection zone in such stars. Accretion rates required to account for the observed abundances of magnesium, 

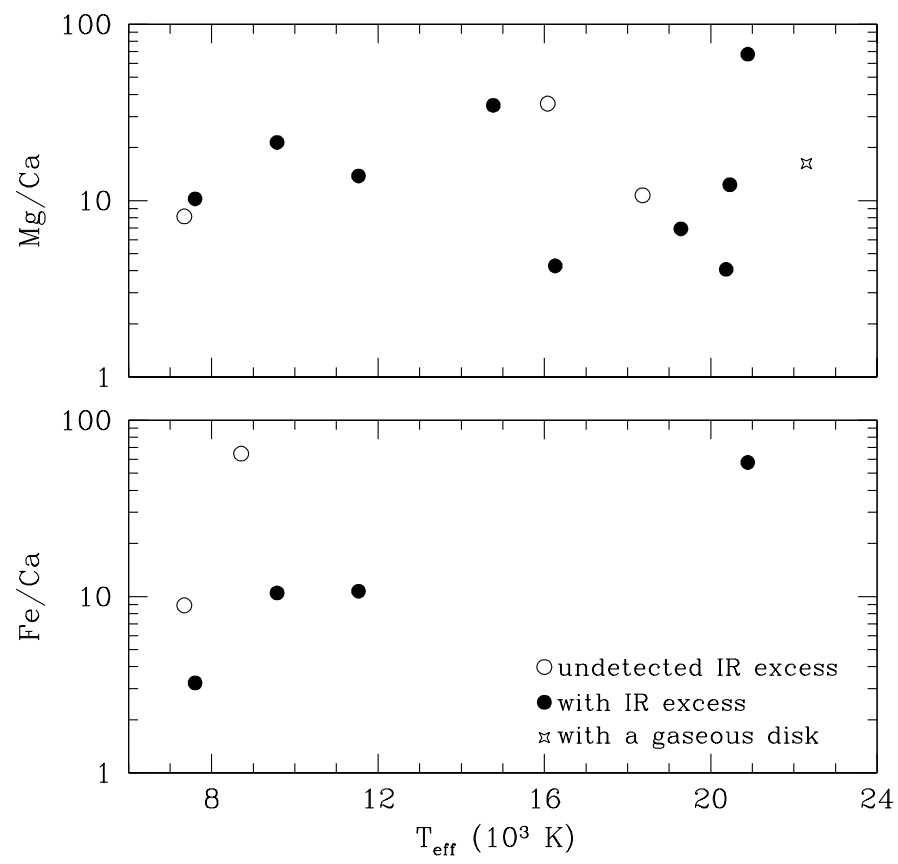

FIGURE 5. The abundance ratios of magnesium (top) and iron (bottom) with respect to calcium.

calcium, or iron are $3 \times 10^{8} \mathrm{~g} \mathrm{~s}^{-1}$ for the hottest white dwarf and $3 \times 10^{9} \mathrm{~g} \mathrm{~s}^{-1}$ for the coolest objects. Details of these calculations and results will be presented elsewhere.

The signal-to-noise of some of the spectra in this study was too low to detect any lines of magnesium, iron, or other elements that may possibly be present in the white dwarf atmosphere. Higher $\mathrm{S} / \mathrm{N}$ spectra at high resolution are required in order to investigate abundance patterns that may be linked to the source of the accreted material.

\section{ACKNOWLEDGMENTS}

This research was supported by GA AV grant numbers IAA301630901 and IAA300030908, and by GA ČR grant number P209/10/0967.

\section{REFERENCES}

Chayer, P., Vennes, S., Pradhan, A. K., Thejll, P., Beauchamp, A., Fontaine, G., \& Wesemael, F. 1995, ApJ, 454, 429

Debes, J.H. 2006, ApJ, 652, 636

Debes, J.H. \& Sigurdsson, S. 2002, ApJ, 572, 556

Dupuis, J., Fontaine, G., \& Wesemael, F. 1993, ApJS, 87, 345

Farihi, J., Jura, M., Lee, J.-E., \& Zuckerman, B. 2010, ApJ, 714, 1386

Farihi, J., Jura, M., \& Zuckerman, B. 2009, ApJ, 694, 805

Gänsicke, B., Marsh, T.R., \& Southworth, J. 2007, MNRAS, 380, L35 
TABLE 1. Atmospheric properties of selected DAZ

\begin{tabular}{|c|c|c|c|c|c|}
\hline Name & $T_{\text {eff }}(\mathbf{K})$ & $\log g$ (c.g.s) & $\log (\mathrm{Ca} / \mathrm{H})$ & $\log (\mathrm{Mg} / \mathrm{H})$ & $\log (\mathrm{Fe} / \mathrm{H}$ \\
\hline WD0032-175 & $9730 \pm 30$ & $8.11 \pm 0.03$ & -9.8 & $\ldots$ & $\ldots$ \\
\hline HS0047+1903 & $16080 \pm 120$ & $7.71 \pm 0.03$ & -7.2 & -5.6 & $\ldots$ \\
\hline HE0106-3253 & $16260 \pm 120$ & $7.93 \pm 0.03$ & -6.6 & -5.9 & $\ldots$ \\
\hline NLTT06390 & $6190 \pm 120$ & $8.39 \pm 0.25$ & -9.6 & $\ldots$ & $\ldots$ \\
\hline WD0208+396 $6^{\mathrm{a}}$ & $7340 \pm 90$ & $8.10 \pm 0.04$ & $-8.8^{\mathrm{b}}$ & -7.9 & $-7.9^{\mathrm{b}}$ \\
\hline WD0243-026 & $6830 \pm 70$ & $8.29 \pm 0.12$ & -9.8 & $\ldots$ & $\ldots$ \\
\hline HS0307+0746 & $10110 \pm 50$ & $7.97 \pm 0.04$ & -8.0 & $\ldots$ & $\ldots$ \\
\hline WD0408-041 & $14770 \pm 100$ & $7.78 \pm 0.02$ & -7.3 & -5.7 & $\ldots$ \\
\hline WD1015+161 & $19280 \pm 150$ & $7.81 \pm 0.03$ & -6.6 & -5.7 & $\ldots$ \\
\hline NLTT23966 & $7960 \pm 100$ & $8.02 \pm 0.14$ & -7.7 & $\ldots$ & $\ldots$ \\
\hline NLTT25792 & $8160 \pm 80$ & $8.12 \pm 0.11$ & -7.3 & $\ldots$ & $\ldots$ \\
\hline WD1102-183 & $8260 \pm 40$ & $8.17 \pm 0.06$ & -10.0 & $\ldots$ & $\ldots$ \\
\hline WD1116+026 & $12450 \pm 220$ & $7.92 \pm 0.06$ & -7.7 & $\ldots$ & $\ldots$ \\
\hline WD1124-293 & $9470 \pm 40$ & $8.11 \pm 0.03$ & -8.8 & $\ldots$ & $\ldots$ \\
\hline WD1150-153 & $11700 \pm 110$ & $7.97 \pm 0.03$ & -7.5 & $\ldots$ & \\
\hline WD1202-232 & $8710 \pm 40$ & $8.18 \pm 0.04$ & -9.8 & $\ldots$ & -8.0 \\
\hline WD1204-136 & $10820 \pm 60$ & $8.18 \pm 0.03$ & -8.3 & $\ldots$ & $\ldots$ \\
\hline HE1225+0038 & $9390 \pm 40$ & $8.07 \pm 0.03$ & -9.8 & $\ldots$ & $\ldots$ \\
\hline HE1315-1105 & $9080 \pm 40$ & $8.09 \pm 0.04$ & -9.4 & $\ldots$ & $\ldots$ \\
\hline WD1337+705 & $19520 \pm 360$ & $7.87 \pm 0.06$ & -6.8 & -5.7 & \\
\hline WD1455+298 & $7600 \pm 160$ & $8.02 \pm 0.26$ & -9.1 & -8.0 & -8.6 \\
\hline WD1457-086 & $20370 \pm 200$ & $7.80 \pm 0.03$ & -6.4 & -5.8 & $\ldots$ \\
\hline WD1614+160 & $16800 \pm 100$ & $7.80 \pm 0.02$ & -7.4 & $\ldots$ & $\ldots$ \\
\hline WD1826-045 & $9010 \pm 30$ & $7.99 \pm 0.04$ & -9.2 & $\ldots$ & $\ldots$ \\
\hline GALEXJ1931+0117 & $20890 \pm 120$ & $7.90 \pm 0.06$ & -6.3 & -4.5 & -4.5 \\
\hline WD2105-820 & $10280 \pm 50$ & $8.01 \pm 0.03$ & -8.7 & $\ldots$ & $\ldots$ \\
\hline WD2115-560 & $9570 \pm 40$ & $8.02 \pm 0.03$ & -8.0 & -6.6 & -6.9 \\
\hline HS2132+0941 & $13060 \pm 140$ & $7.63 \pm 0.04$ & -7.9 & $\ldots$ & $\ldots$ \\
\hline WD2149+021 & $16550 \pm 70$ & $7.87 \pm 0.02$ & -8.0 & $\ldots$ & 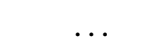 \\
\hline HE2221-1630 & $9990 \pm 40$ & $8.12 \pm 0.04$ & -8.0 & $\ldots$ & $\ldots$ \\
\hline HS2229+2335 & $18360 \pm 140$ & $7.84 \pm 0.03$ & -6.7 & -5.6 & $\ldots$ \\
\hline HE2230-1230 & $19300 \pm 160$ & $7.73 \pm 0.03$ & -7.1 & $\ldots$ & $\ldots$ \\
\hline WD2326+049 & $11530 \pm 80$ & $8.04 \pm 0.03$ & -7.3 & -6.2 & -6.3 \\
\hline
\end{tabular}

${ }^{\mathrm{a}} T_{\text {eff }}, \log g$ from Gianninas et al. (2005)

b From Zuckerman et al. (2003)

c $T_{\text {eff }}, \log g$ determined using SDSS spectroscopy.

d Parameters from Vennes et al. (2010)

Gianninas, A., Bergeron, P., \& Fontaine, G. 2005, ApJ, 631, 1100

Graham, J. R., Matthews, K., Neugebauer, G., \& Soifer, B. T. 1990, ApJ, 357, 216

Holberg, J. B., Barstow, M. A., \& Green, E. M. 1997, ApJ, 474, L127

Jura, M. 2003, ApJ, 584, L91

Kawka, A. \& Vennes, S. 2006, ApJ, 643, 402

Koester, D., Rollenhagen, K., Napiwotzki, R., Voss, B., Christlieb, N., Homeier, D., \& Reimers, D. 2005, A\&A, 432, 1025

Koester, D. \& Wilken, D. 2006, A\&A, 453, 1051

Vennes, S., Kawka, A., \& Németh, P. 2010, MNRAS, 404, L40

Zuckerman, B., Koester, D., Reid, I. N., Hünsch, M. 2003, ApJ, 596, 477 